\title{
Wave emission and absorption at spectral singularities
}

\author{
P. Wang, ${ }^{1}$ L. Jin, ${ }^{1, *}$ G. Zhang, ${ }^{1,2}$ and Z. Song ${ }^{1}$ \\ ${ }^{1}$ School of Physics, Nankai University, Tianjin 300071, China \\ ${ }^{2}$ College of Physics and Materials Science, Tianjin Normal University, Tianjin 300387, China
}

(Received 18 July 2016; published 17 November 2016)

\begin{abstract}
We studied the critical dynamics of spectral singularities. The system investigated is a coupled resonator array with a side-coupled loss (gain) resonator. For a gain resonator, the system acts as a wave emitter at spectral singularities. The reflection probability increased linearly over time. The rate of increase is proportional to the width of the incident wave packet, which served as the spectral singularity observer in the experiment. For a lossy resonator, the system acts as a wave absorber. The emission and absorption states at spectral singularities coalesce in a finite parity-time $(\mathcal{P} \mathcal{T})$ symmetric system that combined by the gain and loss structures cut from corrresponding scattering systems at spectral singularities; in this case, the $\mathcal{P} \mathcal{T}$-symmetric system is at an exceptional point with a $2 \times 2$ Jordan block. The dynamics of the $\mathcal{P} \mathcal{T}$-symmetric system exhibit the characteristic of exceptional points and spectral singularities.
\end{abstract}

DOI: 10.1103/PhysRevA.94.053834

\section{INTRODUCTION}

A non-Hermitian Hamiltonian can possess peculiar features that have no counterpart in a closed Hermitian system. For example, nonreciprocal dynamics have been observed in experiments [1]. Research indicates that the combination of a magnetic field and non-Hermitian potential has an unexpected effect on particle transport behavior [2,3]. The discovery of non-Hermitian Hamiltonians with parity-time $(\mathcal{P} \mathcal{T})$ symmetry and a real spectrum [4] has furthered research on the complex extension of quantum mechanics on a fundamental level [5-18]. In recent years, following revelations of their possible physical relevance by the pioneering work of Ali Mostafazadeh, the spectral singularities of non-Hermitian systems have received considerable attention [19-27]. Most studies have focused on non-Hermitian systems with $\mathcal{P} \mathcal{T}$ symmetry [21,29-38] and non-Hermitian hopping amplitude [39-44].

Spectral singularities are divergences in the continuous spectrum of scattering systems [36] and differ from exceptional points [45-47]. Spectral singularities are attributed to non-Hermitian terms, leading to transmission and (or) reflection coefficients that tend to infinity for an incidence. The steady-states at spectral singularities in scattering systems have the form of propagating plane wave emission and (or) absorption [39]. A spectral singularity can represent lasing with zero linewidth [28,48]. By using Fano resonance in a $\mathcal{P} \mathcal{T}$-symmetric system with a pair of side-coupled balanced gain and loss resonators, spectral singularity induces unidirectional lasing is determined [49], where spectral singularities exhibit nonreciprocity. Complex potential induced spectral singularities in scattering system are intensively investigated [19-21,48-52].

\footnotetext{
*jinliang@nankai.edu.cn

Published by the American Physical Society under the terms of the Creative Commons Attribution 3.0 License. Further distribution of this work must maintain attribution to the author(s) and the published article's title, journal citation, and DOI.
}

Wave packet dynamics at the spectral singularities in a Friedrichs-Fano-Anderson model was predicted [24]. In this paper, we qualitatively studied the dynamic phenomena of wave emission and absorption in the coupled resonator array through a side-coupled gain (loss) resonator using an exact solution of a concrete tight-binding system. Spectral singularities occur when the wave vector matches the coupling strength and gain (loss) rate. The critical behavior associated with the physics of the spectral singularity was investigated, including the wave emission and the perfect absorption of the incident wave. The perfect absorption corresponded to a coherent perfect absorption induced by loss; for wave emission, the reflection probability at the gain resonator increases linearly with time. The secular wave emission forms a platform with wave amplitude being proportional to the full width at half maximum (FWHM) of the incident wave packet. This can serve as the spectral singularity witness in the experiment. We combined the leads of two semi-infinite scattering systems into a $\mathcal{P} \mathcal{T}$-symmetric system. We demonstrated that the $\mathcal{P} \mathcal{T}$-symmetric system was at an exceptional point with a $2 \times 2$ Jordan block when the two semi-infinite scattering systems were at spectral singularities. Thus, we linked the spectral singularity to the exceptional point. We calculated the dynamics of a Gaussian wave in the $\mathcal{P} \mathcal{T}$-symmetric system. A quadratic probability increase was seen for the long-time scale. For the short-time interval, the probability revealed the emission and absorption behavior of the spectral singularities.

This study is organized as follows. In Sec. II, we present the model setup and the solutions. In Sec. III, the spectral singularity of the Hamiltonian is discussed. In Secs. IV and $\mathrm{V}$, we qualitatively analyze the wave emission and perfect absorption of a Gaussian wave packet at the spectral singularities. The scaling law of a wave emission is investigated in detail. In Sec. VI, we compose a $\mathcal{P} \mathcal{T}$-symmetric system at the exceptional point using two semi-infinite scattering systems at the spectral singularities. We demonstrate that the spectral singularities absorption and emission states coalesce in the $\mathcal{P} \mathcal{T}$-symmetric system. Quadratic increases of the wave probability in the $\mathcal{P} \mathcal{T}$-symmetric system are shown to reflect that the system is at the exceptional point, consisting of a $2 \times 2$ Jordan block. Finally, we present a summary in Sec. VIII. 

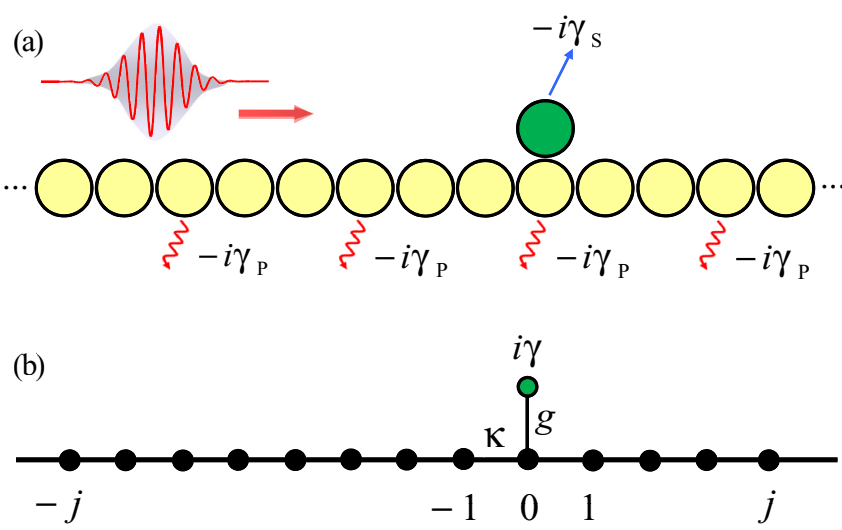

(c)

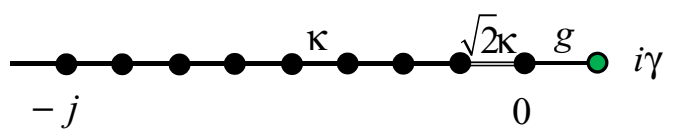

FIG. 1. (a) Schematic configuration for the coupled resonator system. An array of coupled single-mode resonators with a resonator side-coupled at the center. Initially, a photon wave packet moves from the left to the center. Each resonator on the chain array has a decay rate $\gamma_{P}$ and the side-coupled resonator has a decay rate $\gamma_{S}$. (b) Schematic illustration of an equivalent system with a discrete tight-binding model. A tight-binding chain with uniform coupling, side-coupled to a resonator with an imaginary on-site potential $i \gamma$. (c) After symmetric and antisymmetric combination of the uniform chain, we obtained a semi-infinite chain array with gain (loss) resonator coupled at the end.

\section{MODEL}

A one-dimensional coupled resonator array, consisting of a side-coupled resonator is schematically illustrated in Fig. 1(a). The dynamics of the system are characterized by the coupled mode theory [49], and the system is equivalently described by the Hamiltonian

$$
\begin{aligned}
H & =H_{0}-g a_{0}^{\dagger} a_{\mathrm{S}}-g a_{\mathrm{S}}^{\dagger} a_{0}+i \gamma a_{\mathrm{S}}^{\dagger} a_{\mathrm{S}}, \\
H_{0} & =-\kappa \sum_{l=-\infty}^{+\infty}\left(a_{l}^{\dagger} a_{l+1}+a_{l+1}^{\dagger} a_{l}\right)-i \gamma_{\mathrm{P}} \sum_{l=-\infty}^{+\infty} a_{l}^{\dagger} a_{l},
\end{aligned}
$$

where $a_{l}\left(a_{l}^{\dagger}\right)$ annihilates (creates) a photon at the $l$ th resonator. $\gamma_{\mathrm{P}}$ and $\gamma_{\mathrm{S}}$ are the dissipation rates of the cavities in the chain array and the side-coupled resonator, respectively. The relative decay rate is set at $\gamma=\gamma_{\mathrm{P}}-\gamma_{\mathrm{S}}$. The chain array is denoted by $H_{0}$. The term $-i \gamma_{\mathrm{P}}$ has an effect of overall decay on the eigenstates of $H_{0}$. On the condition that the overall decay rate is substantially smaller than the photon tunneling rate, i.e., $\gamma_{\mathrm{P}} \ll$ $\kappa$, the overall decay of the amplitudes is negligible in a finite time scale, $t \sim \gamma_{\mathrm{P}}^{-1}$. The side coupling strength is $g$ and the photon hopping strength for the tunneling between the adjacent cavities is $\kappa$. The Hamiltonian $H$ is schematically illustrated in Fig. 1(b). A candidate realization is using the on-chip coupled microresonator array produced in optical systems $[53,54]$. In previous works, the net gain was created in the microresonator produced on silicon wafers by pumping the doped erbium ions, and the additional loss was induced by a chromium-coated tip $[55,56]$.

On the bases of the wave function ansatz and the mirror symmetry of the Hamiltonian $H$, the solution of the Schrödinger equation

$$
H\left|\psi_{k}^{ \pm}\right\rangle=\varepsilon_{k}\left|\psi_{k}^{ \pm}\right\rangle
$$

can be obtained as

$$
\left\langle j \mid \psi_{k}^{+}\right\rangle=\left\{\begin{array}{ll}
\eta_{k} e^{i k j}-\eta_{-k} e^{-i k j}, & (j \leq-1) \\
-\eta_{-k} e^{i k j}+\eta_{k} e^{-i k j}, & (j \geq 1)
\end{array},\right.
$$

and

$$
\left\langle j \mid \psi_{k}^{-}\right\rangle=\sin (k j), \quad(|j| \geq 1),
$$

where \pm represents the parity of the solution, and

$$
\eta_{k}=2 i \kappa \sin k(i \gamma+2 \kappa \cos k)+g^{2} .
$$

The spectrum is $\varepsilon_{k}=-2 \kappa \cos k$, which is always real for a scattering state.

According to the theory of pseudo-Hermitian quantum mechanics [14], a complete biorthonormal set requires the construction of the eigenfunctions of $H^{\dagger}$. Similarly, the solution of the Schrödinger equation

$$
H^{\dagger}\left|\bar{\psi}_{k}\right\rangle=\varepsilon_{k}\left|\bar{\psi}_{k}\right\rangle
$$

can be obtained by taking $\gamma \rightarrow-\gamma$ from $\left\langle j \mid \psi_{k}^{ \pm}\right\rangle$, i.e.,

$$
\left\langle j \mid \bar{\psi}_{k}^{+}\right\rangle=\left\{\begin{array}{cc}
\eta_{-k}^{*} e^{i k j}-\eta_{k}^{*} e^{-i k j}, & (j \leq-1) \\
-\eta_{k}^{*} e^{i k j}+\eta_{-k}^{*} e^{-i k j}, & (j \geq 1),
\end{array}\right.
$$

and

$$
\left\langle j \mid \bar{\psi}_{k}^{-}\right\rangle=\sin (k j), \quad(|j| \geq 1) .
$$

The following equation can readily be confirmed:

$$
\left\langle\bar{\psi}_{k}^{\lambda} \mid \psi_{k^{\prime}}^{\lambda^{\prime}}\right\rangle=C_{k}^{\lambda} \delta_{\lambda \lambda^{\prime}} \delta_{k k^{\prime}}
$$

where $C_{k}^{\lambda}$ is a bounded real function. This indicates that the wave functions can always be renormalized to achieve a biorthogonal set, except when $k=\pi / 2$ and $2 \kappa \gamma= \pm g^{2}$, which implies the collapse of the biorthonormal relation in Eq. (9).

\section{SPECTRAL SINGULARITY}

In this section, we explore the scattering of the system. We show the dynamic features at the spectral singularity [28]. First, we consider the steady-state solution of the system at the point $\left(k_{\mathrm{c}}, \gamma_{\mathrm{c}}\right)$ with $k_{\mathrm{c}}=\pi / 2$, where the wave packet has the fast velocity $2 \kappa$ and propagates without spreading. The critical gain (loss) rate is

$$
\gamma_{\mathrm{c}}=\sigma g^{2} /(2 \kappa)
$$

where $\sigma=1(\sigma=-1)$ is for the gain (loss) case. When $\gamma=$ $\gamma_{\mathrm{c}}$, the following equations of scattering solution,

$$
\left\langle j \mid \psi_{\mathrm{c}}^{+}\right\rangle=\left\{\begin{array}{lc}
e^{-i \sigma \pi j / 2}, & (j \leq-1) \\
e^{i \sigma \pi j / 2}, & (j \geq 1)
\end{array}\right.
$$


and

$$
\left\langle j \mid \bar{\psi}_{\mathrm{c}}^{+}\right\rangle= \begin{cases}e^{i \sigma \pi j / 2}, & (j \leq-1) \\ e^{-i \sigma \pi j / 2}, & (j \geq 1)\end{cases}
$$

have clear physical implications: the non-Hermitian scattering center acts as a source $(\sigma=1)$ or drain $(\sigma=-1)$ of a $k=\pi / 2$ plane wave. To represent the system at spectral singularities according to the transmission and reflection coefficients, we set a Jost solution for input wave $k=\pi / 2$ :

$$
\left\langle j \mid \psi_{k}\right\rangle= \begin{cases}e^{i k j}+r_{k} e^{-i k j}, & (j \leq-1) \\ t_{k} e^{i k j}, & (j \geq 1)\end{cases}
$$

where the transmission and reflection amplitudes are obtained as

$$
r_{k}=-g^{2} / \eta_{k}, t_{k}=r_{k}+1
$$

This indicates that both $t_{k}$ and $r_{k}$ tend to infinity at the spectral singularity as $\eta_{k}=0$, therefore conforming the theorem proved in Ref. [28].

\section{WAVE EMISSION}

This section addresses implications of the spectral singularity from the perspective of wave packet dynamics. In practice, we consider the time evolution of a Gaussian wave packet with central vector $k$ to reflect the plane wave with vector $k_{\mathrm{c}}$ [61]. The Gaussian wave packet is

$$
\Phi(j, 0)=\Omega^{-1 / 2} e^{-\alpha^{2}\left(j-N_{\mathrm{c}}\right)^{2} / 2} e^{-i k j},
$$

where $N_{\mathrm{c}}$ is the Gaussian wave center and $\Omega=\sqrt{\pi} / \alpha$ is the renormalization factor. This factor ensures the Dirac probability of the initial state is unity. The FWHM of the Gaussian wave packet is $\Delta=2 \sqrt{2 \ln 2} / \alpha$. The velocity is $2 \kappa \sin (k)$ in a uniform chain system with coupling $\kappa$.

Hamiltonian $H$ shown in Fig. 1(b) can be further reduced by symmetric and antisymmetric combination of sites $j<0$ and $j>0$, i.e., $a_{l_{ \pm}}=\left(a_{-l} \pm a_{l}\right) / \sqrt{2}$, as shown in Fig. 1(c). The system is an $N+1$-site coupled resonator array, which consists of a uniform coupled resonator with $N=800$ and the coupling strength is $\kappa$. A resonator with additional loss or gain is coupled at the second last end of the uniform array at the strength $\sqrt{2} \kappa$.

For $\gamma=\gamma_{\mathrm{c}}>0$, the resonator at the end is a gain resonator. We consider a Gaussian wave packet with $\alpha=0.02$ and $k=\pi / 2$, which was centered at $N_{\mathrm{c}}=400$ at time $t=0$. The Gaussian wave packet probability out of region $\left[N_{\mathrm{c}}-\Delta, N_{\mathrm{c}}+\right.$ $\Delta$ ] vanishes approximately and the probability within the $2 \Delta$ region around $N_{\mathrm{c}}$ is over $99.9 \%$. As shown in Fig. 2, the profiles of the Gaussian wave at different times are plotted. The wave packet dynamics exhibited a persistent wave emission from the gain resonator, which reflected the steady-state plane wave emission solution of the system at the spectral singularities. The wave packet was shape-preserving and propagated toward the gain resonator at the end of the resonator chain array. At approximate time $t_{0}=\left(N-N_{\mathrm{c}}-\Delta\right) /(2 \kappa) \approx 141 / \kappa$, the wave packet head reached the gain resonator and the wave packet probability started increasing. The wave packet was reflected as a concomitant amplification and finally formed a platform with the probability height $h$ after the time $t_{0}+\Delta / \kappa$,

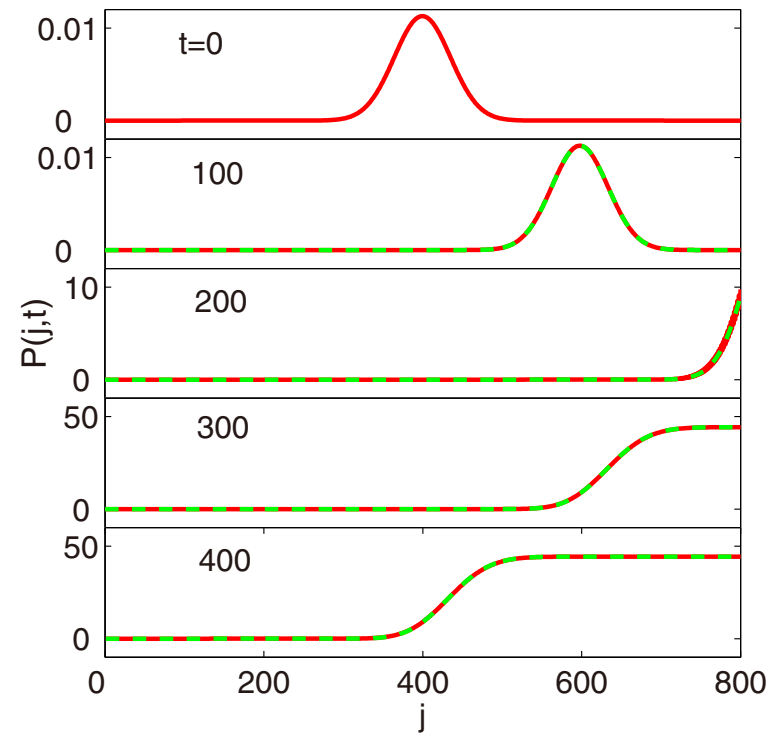

FIG. 2. Scattering process of an incident photon wave packet by the non-Hermitian scattering center. The probability distributions $P(j, t)$ for several instants were obtained according to the time evolution under the systems of the Hamiltonian $H_{0}$ with $\kappa=g=1$ and $\gamma=\gamma_{\mathrm{c}}=\kappa / 2$ at the spectral singularity. The incident wave packet values were $k_{\mathrm{c}}=\pi / 2, N=800, N_{\mathrm{c}}=400$, and $\alpha=0.02$.

characterized by a Gaussian error function that was previously found in systems with complex periodic potentials $[36,60]$. The details are demonstrated in Sec. VI and calculated in Appendices A and B. At the instant $t \gtrsim t_{0}+\Delta / \kappa$, the state $|\Phi(j, 0)\rangle$ evolves to $|\Phi(j, t)\rangle$ and the wave function has the form of

$$
\Phi(j, t)=-\frac{\sqrt{h}}{2}\left\{1+\operatorname{erf}\left[\frac{2^{3 / 4}}{\Delta}\left(j-N_{t}\right)\right]\right\} e^{-\frac{i \pi j}{2}},
$$

where $N_{t}=2(N+1)-N_{\mathrm{c}}-2 \kappa t$ represents the Gaussian wave packet center after reflection for $\gamma=0, h=$ $2\left(\gamma_{\mathrm{c}} / \kappa\right)^{2} \sqrt{\pi} / \alpha$ shows the platform wave emission probability spreading out from the gain resonator to infinity. The wave front of $\Phi(j, t)$ has the velocity $2 \kappa$.
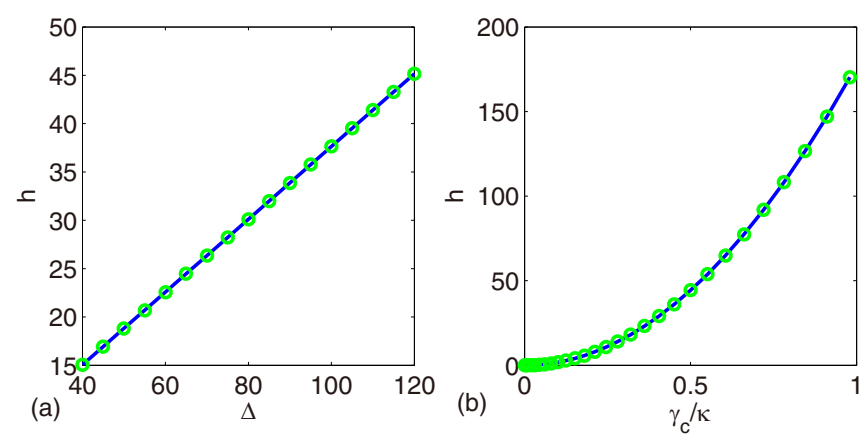

FIG. 3. Scaling of the emission wave probability platform height $h$ as a function of (a) the FWHM $\Delta$ of a Gaussian wave packet at $g / \kappa=1$, and as a function of (b) the resonator gain $\gamma_{\mathrm{c}}$ at the spectral singularities at $\alpha=0.02$. The green circles were obtained through numerical simulation and the blue lines were plots from Eq. (17). 


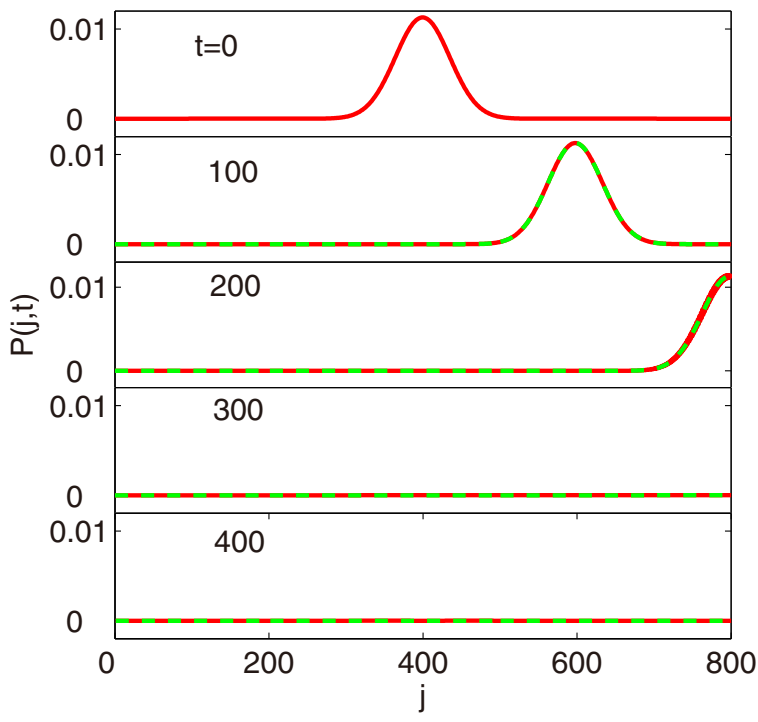

FIG. 4. Scattering process of an incident photon wave packet by the non-Hermitian scattering center. The probability distributions $P(j, t)$ for several instants were obtained according to the time evolution under the systems of the Hamiltonian $H_{0}$ with $\kappa=g=1$ and $\gamma=-\gamma_{\mathrm{c}}=-0.5$ at the spectral singularity. The incident wave packet values were $k_{\mathrm{c}}=\pi / 2, N=800, N_{\mathrm{c}}=400$, and $\alpha=0.02$.

Figure 3(a) shows the scaling of wave-emission platform height $h$ as a function of the FWHM $\Delta$; in Fig. 3(b), the scaling of $h$ as a function of the resonator gain $\gamma$ is shown:

$$
h=\sqrt{\frac{\pi}{2 \ln 2}}\left(\gamma_{\mathrm{c}} / \kappa\right)^{2} \Delta,
$$

where the probability height $h$ linearly depends on the FWHM $\Delta$ and quadratically depends on the resonator gain $\gamma_{c}$ at the spectral singularity.

\section{WAVE ABSORPTION}

For $\gamma=-\gamma_{\mathrm{c}}<0$, the time evolution of $|\Phi(j, 0)\rangle$ differs from the wave emission case $\left(\gamma=\gamma_{\mathrm{c}}>0\right)$. The state $|\Phi(j, 0)\rangle$ will be absorbed at the lossy site. As shown in Fig. 4, a Gaussian wave packet with $k=\pi / 2$, centered at $N_{\mathrm{c}}=400$ at time $t=0$, moves toward the lossy resonator at the end and is absorbed without reflection because of the dissipation. At approximate time $\left(N-N_{\mathrm{c}}-\Delta\right) /(2 \kappa) \approx 141 / \kappa$, the head of the Gaussian wave packet with $\alpha=0.02$ reaches the lossy resonator $N$, and the arrived component is completely absorbed without reflection. At time $t_{0}=\left(N-N_{\mathrm{c}}\right) /(2 \kappa)=$ $200 / \kappa$, the wave packet center arrives at the lossy resonator and half of the wave packet has been perfectly absorbed. After the wave packet moves forward for an extra distance of $\Delta$ with an additional time $\Delta /(2 \kappa)$ cost [i.e., at time $\left.t_{0}+\Delta /(2 \kappa)\right]$, the tail of the wave packet is perfectly absorbed. The absorption demonstrated in the configuration shown in Fig. 1(c) corresponds to the coherent perfect absorption in the configuration of Fig. 1(b). In a coherent perfect absorption, the appropriate amplitudes and phases of the two counterpropagating incidences are perfectly absorbed [57-59]. The wave packet in the configuration depicted in Fig. 1(c) implies

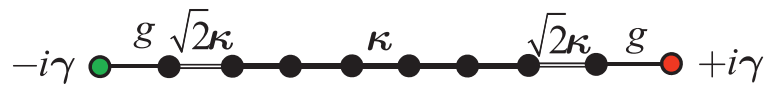

FIG. 5. Schematic configuration of the $\mathcal{P} \mathcal{T}$-symmetric system combining by two semi-infinite scattering systems cut at the leads.

that the two wave packets with the same profile and wave vector that are located at symmetric positions near the lossy resonator $j=0$ in the configuration shown in Fig. 1(b), move in opposite directions, and are perfectly absorbed at the lossy resonator $j=0$.

The time evolution of the initial state $|\Phi(j, 0)\rangle$ is simply a moving Gaussian wave with velocity $2 \kappa$, the center of which is $N_{t}=N_{\mathrm{c}}+2 \kappa t$ before reaching the loss site,

$$
\Phi(j, t)=\Omega^{-1 / 2} e^{-\alpha^{2}\left(j-N_{t}\right)^{2} / 2} e^{-i \frac{\pi}{2} j},
$$

the Gaussian wave packet vanishes out of region $\left[N_{t}-\Delta, N_{t}+\Delta\right]$ because of the exponential decay of the wave packet tails. When the head of the wave packet reaches the lossy resonator and $N_{t}+\Delta>N$, the region $\left[N, N_{t}+\Delta\right]$ is perfectly absorbed but $\left[N_{t}-\Delta, N\right]$ remains. After the tail of the wave packet passes the end of the resonator array when $N_{t}-\Delta>N$, the Gaussian wave packet is completely absorbed.

\section{VI. $\mathcal{P} \mathcal{T}$-SYMMETRIC SYSTEM WITH GAIN AND LOSS}

In this section, we consider a $\mathcal{P} \mathcal{T}$-symmetric system with finite sites (Fig. 5). The finite $\mathcal{P} \mathcal{T}$-symmetric system is formed by connecting a finite gain structure and a finite loss structure. The finite gain (loss) structure is cut at the lead of the semiinfinite chain array for wave emission (perfect absorption) as shown in Fig. 1(c). The $\mathcal{P} \mathcal{T}$-symmetric Hamiltonian can be described by a one-dimensional finite chain array, the ends of which are coupled to two resonators with balanced gain and loss. The Hamiltonian is

$$
\begin{aligned}
H_{\mathcal{P} \mathcal{T}}= & -\kappa\left(\sum_{l=3}^{N-1} a_{l}^{\dagger} a_{l+1}+\sqrt{2} a_{2}^{\dagger} a_{3}+\sqrt{2} a_{N}^{\dagger} a_{N+1}+\text { H.c. }\right) \\
& -g\left(a_{1}^{\dagger} a_{2}+a_{N+1}^{\dagger} a_{N+2}+\text { H.c. }\right) \\
& -i \gamma a_{1}^{\dagger} a_{1}+i \gamma a_{N+2}^{\dagger} a_{N+2} .
\end{aligned}
$$

We consider the $\mathcal{P} \mathcal{T}$-symmetric system as a finite gain structure coupled to its $\mathcal{P} \mathcal{T}$-symmetric loss countpart. Thus, the $\mathcal{P} \mathcal{T}$-symmetric system has even site $N$. The exceptional point is at $\gamma_{\mathrm{c}}=g^{2} / 2 \kappa$ as demonstrated in detail in Appendix A. In weak gain or loss region $|\gamma| \leq \gamma_{\mathrm{c}}$, the system has two pairs of bound states with complex eigenvalues; in strong gain or loss region $|\gamma|>\gamma_{\mathrm{c}}$, one more pair of bound states emerge with pure imaginary eigenvalues. The system is nondiagonalizable consisting of a $2 \times 2$ Jordan block. The coalesced state is a plane wave state that emits from the gain resonator to the loss resonators. The plane wave state is the coalescence of the wave emission state and the absorption state at the spectral singularity of the semi-infinite systems, with gain and loss resonator at the ends.

In Fig. 6, we show a Gaussian wave with $k=\pi / 2$, centered at $N_{\mathrm{c}}=400$, moving with velocity $2 \kappa$ toward the gain resonator. In order to simulate wave packet dynamics, 


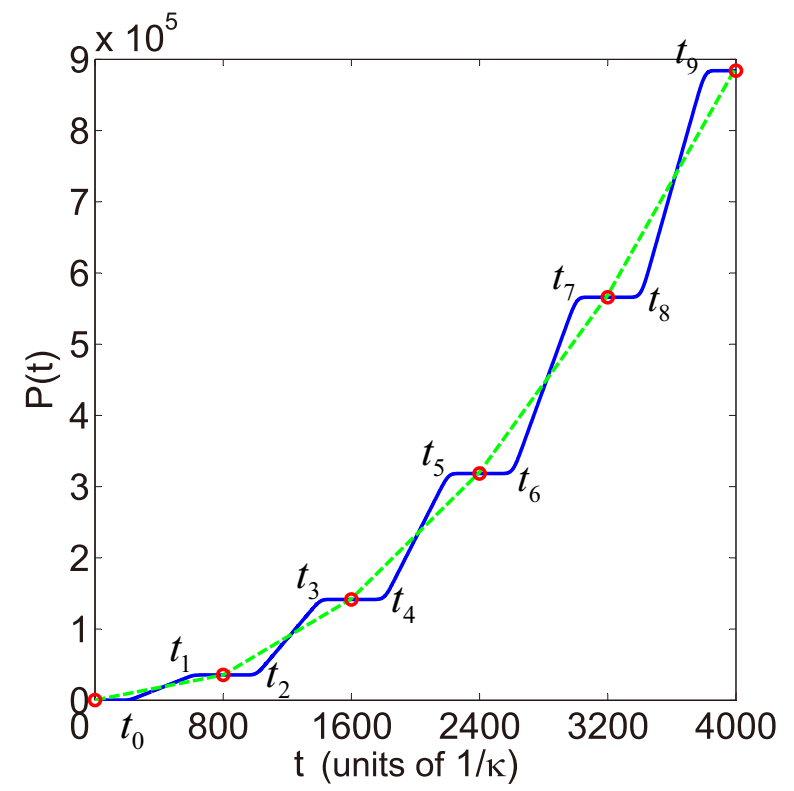

FIG. 6. Time evolution probability of Gaussian wave in a $\mathcal{P} \mathcal{T}$ symmetric finite system (blue curve). Red circles show the probability when the wave front is at the center of the $\mathcal{P} \mathcal{T}$-symmetric system. The green curve indicates the quadratic increasing of wave packet probability in a long time scale. $t_{n}$ marks the time of the wave front reflected at the ends resonator with gain and loss. The parameters are $g=\kappa=1, \alpha=0.02, k=\pi / 2, N=800, N_{\mathrm{c}}=400$.

we omit the significantly small probabilities of wave packet on the two pairs of bound states, which are far away from the wave packet center in the momentum space. At the time $t_{0}=200 / \kappa$ (reaching the system ends for the first time), the wave packet probability starts increasing because it reaches the gain resonator. The emission wave at the gain resonator leads to a linear growth after wave packet reflection. The reflection at the gain resonator realizes a plane wave emission process as discussed in the preceding section. The emission wave still has a velocity of $2 \kappa$ and at time $t_{1}=600 / \kappa$, the wave reaches the loss point and is absorbed. After the wave is reflected at the loss resonator, the waves that are emitted and absorbed are balanced and the probability remains constant until the wave front reaches the gain resonator again at $t_{2}=1000 / \kappa$. In the time interval $\left[t_{1}, t_{2}\right]$, the wave emission at the gain resonator and wave absorption at the loss resonator are balanced. The wave increases again at the time interval $\left[t_{2}, t_{3}\right]$. After the wave front touches the loss resonator at $t_{3}=1400 / \kappa$, the dynamics of the right propagating wave with constant probability and the left propagating wave with a linear time-dependent probability growth repeat as described. $t_{n}=t_{0}+m N /(2 \kappa)(m \in \mathbb{Z})$ is the time wave reflection happens at the resonators with gain and loss. The dynamics of $2 \times 2$ Jordan blocks for dimer systems have been thoroughly investigated. The probability in the dimer exhibits an increasing power law with the highest order being a quadratic function [62-64]. The increasing quadratic is associated with the $2 \times 2$ Jordan block. For a higher-order Jordan block (e.g., a $3 \times 3$ Jordan block), the highest order of the probability increase is higher (quartic function). We note that in the $\mathcal{P} \mathcal{T}$-symmetric system, the wave packet probability increases quadratically in a large time scale,

$$
P(t) \approx 1+(h / N)(\kappa t)^{2},
$$

as denoted by the dashed green line shown in Fig. 6, where the coefficient $h / N \approx 1 / 18$. The red circles in Fig. 6 indicate the probabilities of the wave at the chain center at time $m N / \kappa(m \in$ $\mathbb{Z}$ ). The quadratic probability increase reflects that the system is at the exceptional point. The Hamiltonian system consists of a $2 \times 2$ Jordan block with two coalescence states. Thus, we link the spectral singularities of a semi-infinite scattering system with the exceptional point of a finite $\mathcal{P} \mathcal{T}$-symmetric system.

In the Appendix, we calculate the dynamics of a Gaussian wave packet in the $\mathcal{P} \mathcal{T}$-symetric system. In a large system that the wave packet width far less than the system size ( $\Delta \ll N$ ), the wave packet is localized without spreading in the dynamical process before the wave front reach the system ends. The system has no long-range interactions and is described by a tight-binding model. The loss far away from the gain in the system hardly affects the dynamics of a wave packet that close to the area near the gain site, therefore, the dynamics in the $\mathcal{P} \mathcal{T}$-symmetric system is approximately the same as that in the corresponding wave emission system.

\section{NEAR THE SPECTRAL SINGULARITIES}

The characteristic dynamics at and near the system exceptional points was investigated in several previous works [64,65]. The polynomial dynamical features at the exceptional points were also found near the exceptional points in a narrow region. In this section, we show the dynamical behaviors near the spectral singularities. The reflection coefficient for $k=\pi / 2$ wave in configuration Fig. 1(c) is $r=\left(2 \gamma+g^{2}\right) /\left(2 \gamma-g^{2}\right)$. For $\gamma>0(\gamma<0)$, the reflection is larger (smaller) than unity, which is attributed to the gain (loss) at the system end. The reflection probability $R(\gamma)=|r|^{2}$ is shown in Figs. 7(a) and 7(b). The reflection coefficient diverges at the spectral singularities for system with gain, and changes dramatically near the spectral singularities [Fig. 7(a)]. For system with loss, the reflection coefficient is the inverse, i.e., $R(-\gamma)=R(\gamma)^{-1}$, the reflection at the spectral singularities is zero. Contrary to the system with gain, the reflection is insensitive as the loss $\gamma$ near the spectral singularities, the reflection probability is close to zero in a wide region near the spectral singularities, in particular at large $\gamma_{\mathrm{c}}$ [Fig. 7(b)].

In order to investigate the dynamics near the spectral singularities, we define the gain or loss $\gamma$ deviated from the spectral singularities by a deviation parameter $\delta$ as

$$
\gamma=\gamma_{\mathrm{c}}(1+\delta)
$$

The dynamical difference is enlarged as time increasing for $\gamma>0$. We focus on a short time internal within the wave packet reaching the gain site for the second time as shown in Figs. 7(c)-7(f). In Figs. 7(c) and 7(d), we show the dynamical time evolution of the probability of an incident wave packet at $g=\kappa=1$. At about $t=300 / \kappa$ in Fig. 7(c), the wave emission starts, the probability increases linearly at the spectral singularity. For the gain $\gamma$ slightly deviates from the critical gain $\gamma_{\mathrm{c}}$ at about $\delta=10^{-4}$, the time evolution keeps close with the linear increasing in a time interval before the wave packet 


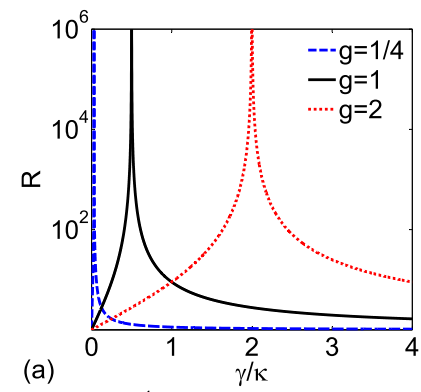

(a)
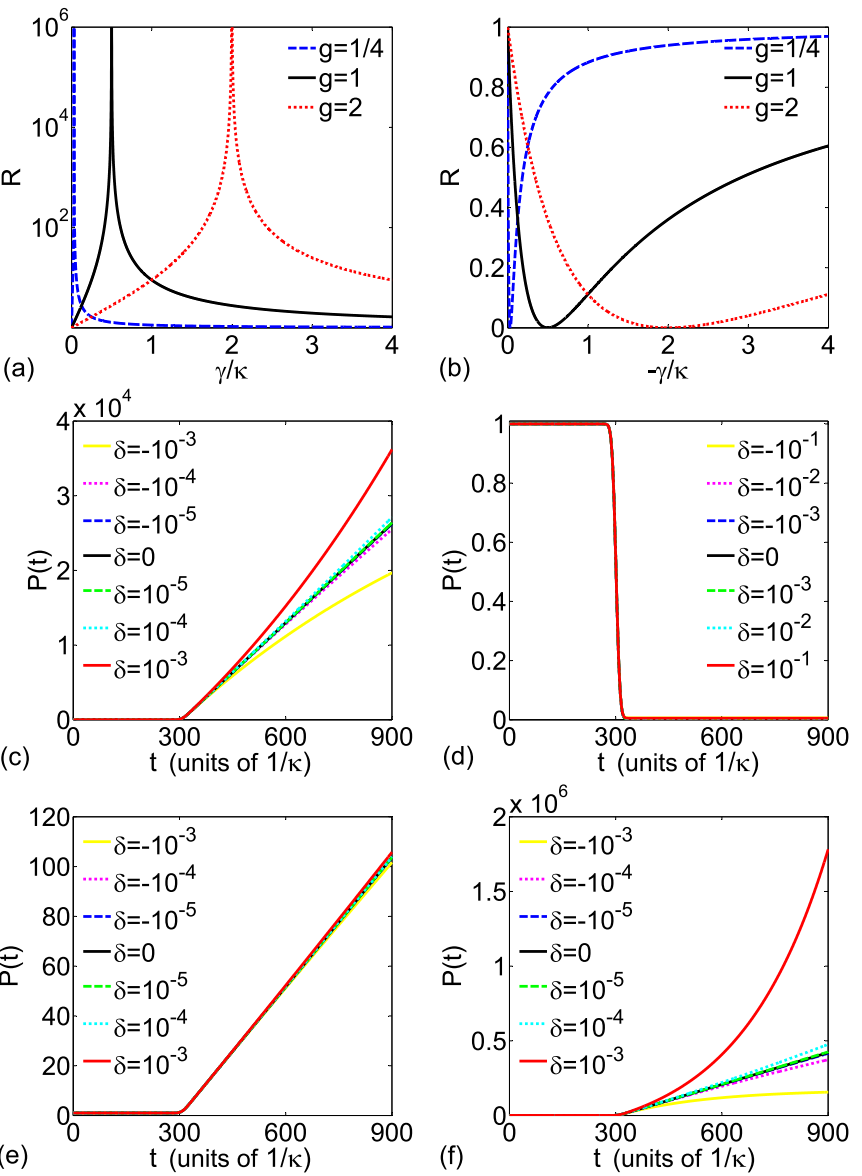

(e)

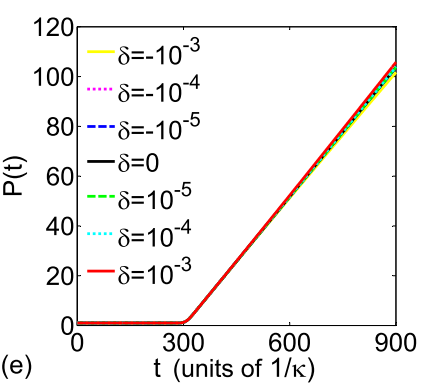

FIG. 7. The reflection probability and the dynamics near the spectral singularities for the configuration Fig. 1(c). The reflection $R(\gamma)$ for gain (a) and loss (b). (c-f) The curves correspond to systems with different deviations $\delta$ in the gain (c, e, f) or loss (d). The parameters are $\kappa=1, \alpha=0.04, k=\pi / 2, N=800, N_{\mathrm{c}}=200$; $g=1$ in (c, d), $g=1 / 4$ in (e), $g=2$ in (f).

reaching the gain site once more after reflection. We consider the probability difference $\left(\left|1-P_{\gamma} / P_{\gamma_{\mathrm{c}}}\right|\right)$ at $t=900 / \kappa$. The probability difference remains within $3 \%$ for $|\delta| \leq 10^{-4}$, the probability difference for $|\delta|=10^{-3}$ is remarkable, reaches about $40 \%$. In Fig. 7(d), the perfect absorption in a loss system remains good for large deviation. From the expression of reflection $R(\gamma)$, the probability without absorption is around $1 \%$ for loss deviation as large as $|\delta|=10^{-1}$. After the wave packet reached the loss site for the first time (around $t=300 / \kappa)$, the wave packet is almost fully absorbed.

In Figs. 7(e) and 7(f), we show the time evolution of probability at different spectral singularities. The spectral singularities at different critical gain $\gamma_{\mathrm{c}}$ varies as the coupling $g$. Strong coupling $g$ results in large gain $\gamma_{\mathrm{c}}$. The probability difference in the time evolution process is gain dependent, the significant probability difference is caused by the large gain. As the critical gain reduced to $\gamma_{\mathrm{c}}=\kappa / 32$ at $g=\kappa / 4$, the probability difference decreases to $2 \%$ for $\delta=10^{-3}$ [Fig. 7(e)]; as the critical gain increased to $\gamma_{\mathrm{c}}=2 \kappa$ at $g=2 \kappa$, the probability difference increases to $320 \%$ for $\delta=10^{-3}$ [Fig. 7(f)]. Moreover, through comparison, we note that the probability difference is smaller for negative $-|\delta|$ than that of

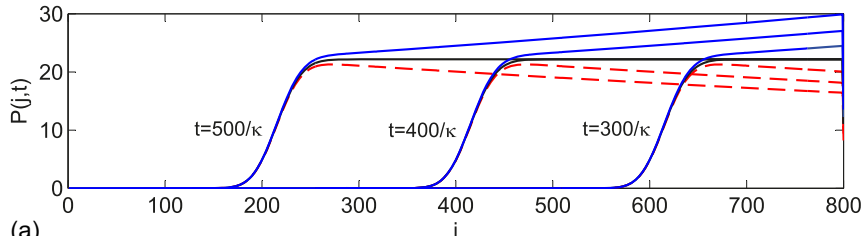

(a)
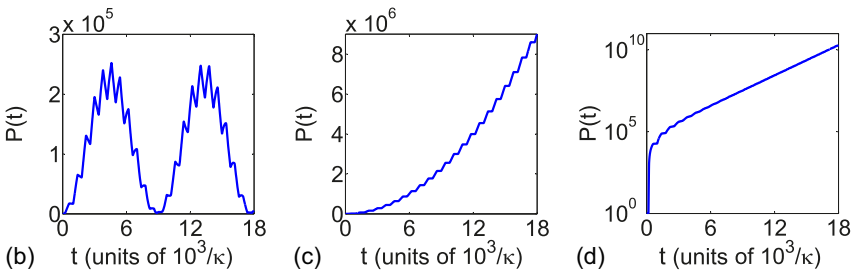

FIG. 8. The probabilities of Gaussian wave packet in the systems near the spectral singularities. $g=\kappa=1, \alpha=0.04, k=\pi / 2, N=$ $800, N_{\mathrm{c}}=400$. (a) $|\delta|=0,10^{-3}$ for the scattering systems; (b) $\delta=$ $-10^{-4}$, (c) $\delta=0$, (d) $\delta=10^{-4}$ for the $\mathcal{P} \mathcal{T}$-symmetric systems.

positive $|\delta|$, which also indicates that large gain leading to more probability difference. For a system with loss, the absorption is insensitive near the spectral singularities in a wide region, in particular for systems with large loss.

In Fig. 8(a), the time evolution profiles of Gaussian wave packet in the scattering system as illustrated in Fig. 1(c) are shown for $|\delta|=0,10^{-3}$. The red dashed (blue solid) lines are for $\delta=-10^{-3}\left(\delta=10^{-3}\right)$, they are similar but slight smaller (larger) than the platform wave emission at the spectral singularity (black solid lines), the profiles deviations diminish as $|\delta|$ decrease. In Figs. 8(b)-8(d), the time evolution of probabilities in the $\mathcal{P} \mathcal{T}$-symmetric system (Fig. 5) are shown for different deviations $\delta$. The probability oscillates in the region $\gamma<\gamma_{\mathrm{c}}$ in Fig. 8(b), quadratically increases at the exceptional point in Fig. 8(c), and exponentially increases in the region $\gamma>\gamma_{\mathrm{c}}$ in Fig. 8(d). The exponential increase is induced by the one more pair of bound states emerged with pure imaginary eigenvalues when $\gamma>\gamma_{\mathrm{c}}$.

\section{CONCLUSION}

We studied the spectral singularity of a semi-infinite coupled resonator system. The system is a coupled resonator array with a gain (lossy) resonator at the end. This system can be described by a one-dimensional tight-binding chain. Based on the exact solution, the critical dynamical behavior associated with the spectral singularities was demonstrated. The dissipative resonator absorbs the incoming wave with a matched wave vector. When the resonator loss is replaced by a gain, the coupled resonator system remains at the spectral singularity. However, the dynamics is a persistent wave emission instead of a perfect absorption. We found that the wave emission can be characterized by a time-dependent Gaussian error function. The emission wave amplitude probability linearly depends on the FWHM of the incident Gaussian wave and quadratically depends on the resonator gain. Combination of the scattering systems at spectral singularities can form a finite $\mathcal{P} \mathcal{T}$-symmetric system at exceptional point. Thus, we linked the spectral singularity to the exceptional point. The coalescence states are the absorption and emission 
solutions at the spectral singularities in the scattering system. The occurrence of two coalescent states indicates that the $\mathcal{P} \mathcal{T}$-symmetric system has a $2 \times 2$ Jordan block and that the probability increases quadratically. Calculating the time evolution of Gaussian wave packet with energy on resonance of the coalesced states, we revealed a stepped progressive ascent of probability. For a long time scale, the probability increases quadratically, which reflects the exceptional point; for a short time scale, the plane wave emission and absorption phenomena reflect the dynamics at the spectral singularities. The dynamical features near the spectral singularities were investigated, the dynamical differences in the wave packet profiles and probabilities induced by slight deviation from the critical gain (loss) was shown, the effect bringing by the deviation is significant (tiny) at large critical gain (loss).

\section{ACKNOWLEDGMENTS}

We acknowledge the support of CNSF (Grants No. 11605094 and No. 11374163) and the Baiqing Plan of Nankai University (Grant No. ZB15006104).

\section{APPENDIX A: SOLUTION OF THE $\mathcal{P} \mathcal{T}$-SYMMETRIC SYSTEM}

We consider the $\mathcal{P} \mathcal{T}$-symmetric Hamiltonian Eq. (19). Introducing a local unitary transformation $u$, which is defined by $u a_{j}^{\dagger} u^{-1}=(-1)^{j} a_{j}^{\dagger}, u a_{j} u^{-1}=(-1)^{j} a_{j}$, we have $(\mathcal{P} u) H(\mathcal{P} u)^{-1}=-H$, i.e., $H$ has a chiral symmetry. On the other hand, unitary transformation $\mathcal{P} u$ maintains the spectrum of the system. Then the spectrum is symmetric about zero energy. From the investigation of the zero-energy solution, we obtain one zero-energy state for system with odd $N$, and two coalesced zero-energy states at the exceptional point $\gamma_{\mathrm{c}}=$ $g^{2} /(2 \kappa)$ for system with even $N$ (the situation we discussed in Sec. VI: The $\mathcal{P} \mathcal{T}$-symmetric system is a finite gain structure coupled to its $\mathcal{P} \mathcal{T}$-symmetric loss structure counterpart). form

In general, the single-particle solution for any $N$ has the

$$
\left|\varphi_{k}\right\rangle=\sum_{l} f_{l}|l\rangle,
$$

the wave function ansatz is $f_{l}=A e^{i k l}+B e^{-i k l}$ for the uniform region of the system $(3 \leq l \leq N)$. To be concise, $\sum_{l}$ represents the summation of all $l$ ranging from 1 to $N+2$. The Schrödinger equation can be expressed in the explicit form of

$$
\begin{gathered}
-g f_{2}=\left(E_{k}+i \gamma\right) f_{1}, \\
-g f_{1}-\sqrt{2} \kappa f_{3}=E_{k} f_{2}, \\
-\sqrt{2} \kappa f_{2}-\kappa f_{4}=E_{k} f_{3}, \\
-\kappa f_{N-1}-\sqrt{2} \kappa f_{N+1}=E_{k} f_{N}, \\
-g f_{N+2}-\sqrt{2} \kappa f_{N}=E_{k} f_{N+1}, \\
-g f_{N+1}=\left(E_{k}-i \gamma\right) f_{N+2},
\end{gathered}
$$

where $E_{k}=-2 \kappa \cos k$ is the eigen energy. Substituting $f_{l}=$ $A e^{i k l}+B e^{-i k l}(3 \leq l \leq N)$, we obtain

$$
\begin{gathered}
-g f_{2}-\left(E_{k}+i \gamma\right) f_{1}=0, \\
-g f_{1}-\sqrt{2} \kappa\left(A e^{3 i k}+B e^{-3 i k}\right)-E_{k} f_{2}=0, \\
-\sqrt{2} \kappa f_{2}-\left(E_{k} e^{3 i k}+\kappa e^{4 i k}\right) A-\left(E_{k} e^{-3 i k}+\kappa e^{-4 i k}\right) B=0, \\
-\sqrt{2} \kappa f_{N+1}-\left(E_{k} e^{i k N}+\kappa e^{i k(N-1)}\right) A \\
-\left(E_{k} e^{-i k N}+\kappa e^{-i k(N-1)}\right) B=0, \\
-g f_{N+2}-\sqrt{2} \kappa\left(A e^{i k N}+B e^{-i k N}\right)-E_{k} f_{N+1}=0, \\
-g f_{N+1}-\left(E_{k}-i \gamma\right) f_{N+2}=0 ; \\
\text { (A12) } \\
\text { after eliminating the } \operatorname{coefficients} \text { of wave function, the wave } \\
\text { vector } k \operatorname{satisfies}^{2} \\
\left.4 \kappa^{2}\left[\kappa^{2} \sin ^{2}(2 k)+\gamma^{2} \sin ^{2} k\right]-g^{4}\right\} \sin [(N-1) k] \\
+4 g^{2} \kappa^{2} \sin (2 k) \cos [(N-1) k]=0 .
\end{gathered}
$$

It is easy to check that $k=\pi / 2$ is a solution of Eq. (A14) for arbitrary system parameters with odd $N$; but the parameters should satisfy $\gamma=g^{2} /(2 \kappa)$ for system with even $N$.

Now we consider the wave vector $k=\pi / 2$. The corresponding wave function $\left|\varphi_{\pi / 2}\right\rangle$ at $\gamma=g^{2} /(2 \kappa)$ (i.e., $E_{k}=0$ ) under the Schrödinger equations of Hamiltonian $H$ yields

$$
\begin{gathered}
-f_{2}=i g /(2 \kappa) f_{1}, \\
-g f_{1}=\sqrt{2} \kappa(-i A+i B), \\
-\sqrt{2} f_{2}=A+B, \\
-\sqrt{2} f_{N+1}=i^{N-1} A+(-i)^{N-1} B, \\
-g f_{N+2}=\sqrt{2} \kappa\left[i^{N} A+(-i)^{N} B\right],
\end{gathered}
$$

where we obtain $A=0, B=-\sqrt{2} f_{2}$, and

$$
\begin{gathered}
f_{1}=(2 i \kappa / g) f_{2}, \\
f_{l}=-\sqrt{2}(-i)^{l} f_{2},(3 \leq l \leq N), \\
f_{N+1}=(-i)^{N-1} f_{2}, \\
f_{N+2}=(-i)^{N}(2 \kappa / g) f_{2} .
\end{gathered}
$$

Note that $A=0$ indicates that $\left|\varphi_{\pi / 2}\right\rangle$ is a unidirectional plane wave with left-going part $e^{-i k j}$ only.

Similarly, the wave function $\left|\tilde{\varphi}_{\pi / 2}\right\rangle$ of Hamiltonian $H^{\dagger}$ for $k=\pi / 2$ at $\gamma=g^{2} /(2 \kappa)$ under the Schrödinger equations yields

$$
\begin{gathered}
-\tilde{f}_{2}=-i g /(2 \kappa) \tilde{f}_{1}, \\
-g \tilde{f}_{1}=\sqrt{2} \kappa(-i \tilde{A}+i \tilde{B}),
\end{gathered}
$$




$$
\begin{gathered}
-\sqrt{2} \tilde{f}_{2}=\tilde{A}+\tilde{B}, \\
-\sqrt{2} \tilde{f}_{N+1}=i^{N-1} \tilde{A}+(-i)^{N-1} \tilde{B}, \\
-g \tilde{f}_{N+2}=\sqrt{2} \kappa\left[i^{N} \tilde{A}+(-i)^{N} \tilde{B}\right],
\end{gathered}
$$

where we obtain $\tilde{A}=-\sqrt{2} \tilde{f}_{2}, \tilde{B}=0$, and

$$
\begin{gathered}
\tilde{f}_{1}=-(i 2 \kappa / g) \tilde{f}_{2}, \\
\tilde{f}_{l}=-\sqrt{2} i^{l} \tilde{f}_{2},(3 \leq l \leq N), \\
\tilde{f}_{N+1}=i^{N-1} \tilde{f}_{2}, \\
\tilde{f}_{N+2}=i^{N}(2 \kappa / g) \tilde{f}_{2},
\end{gathered}
$$

therefore, the overlap is $\left\langle\tilde{\varphi}_{\pi / 2} \mid \varphi_{\pi / 2}\right\rangle=\sum_{l} \tilde{f}_{l}^{*} f_{l}$, i.e.,

$$
\begin{aligned}
\left\langle\tilde{\varphi}_{\pi / 2} \mid \varphi_{\pi / 2}\right\rangle= & -\frac{4 \kappa^{2}}{g^{2}}+1+2 \sum_{l=3}^{N}(-1)^{l}-(-1)^{N} \\
& +(-1)^{N} \frac{4 \kappa^{2}}{g^{2}}
\end{aligned}
$$

for even $N,\left\langle\tilde{\varphi}_{\pi / 2} \mid \varphi_{\pi / 2}\right\rangle=0$; for odd $N,\left\langle\tilde{\varphi}_{\pi / 2} \mid \varphi_{\pi / 2}\right\rangle=$ $-8 \kappa^{2} / g^{2} \neq 0$. These indicate that $\gamma=g^{2} /(2 \kappa)$ is the exceptional point of the $\mathcal{P} \mathcal{T}$-symmetric system with even $N$, the coalescence states are the zero-energy states; however, $\gamma=g^{2} /(2 \kappa)$ is not the exceptional point of the $\mathcal{P} \mathcal{T}$-symmetric system with odd $N$.

The time evolution of a Gaussian wave packet with central momentum $\pi / 2$ is relevant to the near-zero-energy eigen states, i.e., $k \approx \pi / 2$, in which Eq. (A14) under similar coupling condition $(\kappa \sim g)$ is approximately reduced into

$$
\tan (N k) \approx 0,
$$

the approximate solution of momentum $k$ near $\pi / 2$ is

$$
k \approx n \pi / N \text {. }
$$

\section{APPENDIX B: DYNAMICS IN THE $\mathcal{P} \mathcal{T}$-SYMMETRIC SYSTEM}

We apply the solution of $\mathcal{P} \mathcal{T}$-symmetric system with odd $N$ to calculate the dynamics of a Gaussian wave packet. The gain $\gamma=g^{2} /(2 \kappa)$ is not the exceptional point of $\mathcal{P} \mathcal{T}$-symmetric system with odd $N$, thus the biorthoganal basis exists for $H$ and $H^{\dagger}$. We solve the eigen states of $H$ and $H^{\dagger}$ to form the biorthogonal basis. We use the biorthoganal basis to expand the initial state and calculate the time evolution. From Eqs. (A8)(A10), we obtain $f_{1}, A$, and $B$ as functions of $f_{2}$,

$$
\begin{gathered}
f_{1}=\frac{g}{2 \kappa \cos k-i \gamma} f_{2}, \\
A=\frac{2 i \kappa^{2} \sin (2 k)+2 \kappa \gamma \sin k-g^{2}}{2 i \kappa \sqrt{2} e^{2 i k}(2 \kappa \cos k-i \gamma) \sin k} f_{2}, \\
B=\frac{2 i \kappa^{2} \sin (2 k)+2 \kappa \gamma \sin k+g^{2}}{2 i \kappa \sqrt{2} e^{-2 i k}(2 \kappa \cos k-i \gamma) \sin k} f_{2} ;
\end{gathered}
$$

the coefficients $f_{N+1}, f_{N+2}$ can be obtained as functions of $f_{2}$ after substituting $A, B$ in Eqs. (A11)-(A13), $H^{\dagger}$ is solved under the same procedure. The eigen states for $H^{\dagger}$ are set as $\left|\tilde{\varphi}_{k}\right\rangle=\sum_{l} \tilde{f}_{l}|l\rangle$ with $\tilde{f}_{l}=\tilde{A} e^{i k l}+\tilde{B} e^{-i k l}(3 \leq l \leq N)$, similarly, we obtain

$$
\begin{gathered}
\tilde{f}_{1}=\frac{g}{2 \kappa \cos k+i \gamma} \tilde{f}_{2}, \\
\tilde{A}=\frac{2 i \kappa^{2} \sin (2 k)-2 \kappa \gamma \sin k-g^{2}}{2 i \kappa \sqrt{2} e^{2 i k}(2 \kappa \cos k+i \gamma) \sin k} \tilde{f}_{2}, \\
\tilde{B}=\frac{2 i \kappa^{2} \sin (2 k)-2 \kappa \gamma \sin k+g^{2}}{2 i \kappa \sqrt{2} e^{-2 i k}(2 \kappa \cos k+i \gamma) \sin k} \tilde{f}_{2},
\end{gathered}
$$

the coefficients $\tilde{f}_{N+1}, \tilde{f}_{N+2}$ can be obtained as functions of $\tilde{f_{2}}$. All coefficients $A, B, \tilde{A}, \tilde{B}$ and wave functions $f_{1}, f_{N+1}$, $f_{N+2}, \tilde{f}_{1}, \tilde{f}_{N+1}, \tilde{f}_{N+2}$ are expressed as functions of nonzero values $f_{2}, \tilde{f}_{2}$. The momentum $k$ can be solved from the critical Eq. (A14) exactly; however, the eigen states evolved in the time evolution process and being important are the eigen states in the linear region of the spectrum near $k=\pi / 2$, which has approximate solution of $k \approx n \pi / N$.

Note that the wave functions $\left|\varphi_{k}\right\rangle$ and $\left|\tilde{\varphi}_{k}\right\rangle$ should be renormalized. In a non-Hermitian system, we use the biorthogonal renormalization method. The renormalization factors are $G_{k}=\left\langle\tilde{\varphi}_{k} \mid \varphi_{k}\right\rangle=\sum_{l} \tilde{f}_{j}^{*} f_{j}$. The renormalized eigen states of Hamiltonians $H$ and $H^{\dagger}$ are denoted as

$$
\begin{aligned}
\left|\varphi_{G_{k}}\right\rangle & =G_{k}^{-1 / 2} \sum_{l} f_{l}|l\rangle, \\
\left|\tilde{\varphi}_{G_{k}}\right\rangle & =G_{k}^{*-1 / 2} \sum_{l} \tilde{f}_{l}|l\rangle,
\end{aligned}
$$

for eigenvalues $E_{k}$ and $E_{k}^{*}$, respectively. The overlap is $\left\langle\tilde{\varphi}_{G_{k}} \mid \varphi_{G_{k}}\right\rangle=1$ according to the definition of $G_{k}$.

The initial state is a Gaussian wave packet with central momentum $\pi / 2$, centered at site $N_{\mathrm{c}}$,

$$
|\psi(0)\rangle=\Omega^{-1 / 2} \sum_{j} e^{-\alpha^{2}\left(j-N_{\mathrm{c}}\right)^{2} / 2} e^{i(\pi / 2) j}|j\rangle,
$$

the time evolution of the initial state is in form of $|\psi(t)\rangle=$ $e^{-i H t}|\psi(0)\rangle$, which can be calculated by employing the biorthogonal basis $\left\{\left|\varphi_{G_{k}}\right\rangle,\left|\tilde{\varphi}_{G_{k}}\right\rangle\right\}$ that composed by the eigen states of Hamiltonians $H$ and $H^{\dagger}$ [Eqs. (B7) and (B8)]. $|\psi(t)\rangle$ in the momentum space of $H$ is in form of

$$
|\psi(t)\rangle=\sum_{k} e^{-i E_{k} t}\left\langle\tilde{\varphi}_{G_{k}} \mid \psi(0)\right\rangle\left|\varphi_{G_{k}}\right\rangle .
$$

The Gaussian wave packet is localized in both real and momentum spaces; a wide Gaussian wave packet in the real space is narrow in the momentum space. For the Gaussian wave packet with central momentum $\pi / 2$, the relevant eigen states that being important in the time evolution process are the states with momentum $k$ near $\pi / 2$. Therefore, to calculate the dynamical factor $e^{-i E_{k} t}$, we can approximately express the eigen energy near momentum $k_{\mathrm{c}}=\pi / 2$,

$$
E_{k}=-2 \kappa \cos k \approx 2 \kappa\left(k-k_{\mathrm{c}}\right) .
$$

Substituting the expansion coefficients $\left\langle\tilde{\varphi}_{G_{k}} \mid \psi(0)\right\rangle$, the time evolution state $|\psi(t)\rangle$ is in form of

$$
\begin{aligned}
|\psi(t)\rangle= & \frac{1}{\sqrt{\Omega}} \sum_{k} \frac{1}{\sqrt{G_{k}}}\left(\sum_{j} \tilde{f}_{j}^{*} e^{-\frac{\alpha^{2}\left(j-N_{\mathrm{c}}\right)^{2}}{2}} e^{i k_{\mathrm{c}} j}\right) \\
& \times e^{-i 2 \kappa t\left(k-k_{\mathrm{c}}\right)}\left|\varphi_{G_{k}}\right\rangle
\end{aligned}
$$




$$
\begin{aligned}
& \approx \frac{1}{\sqrt{\Omega}} \sum_{k} \frac{\tilde{A}^{*}}{\sqrt{G_{k}}}\left(\sum_{j} e^{-i k j} e^{-\frac{\alpha^{2}\left(j-N_{\mathrm{c}}\right)^{2}}{2}} e^{i k_{\mathrm{c}} j}\right) \\
& \times e^{-i 2 \kappa t\left(k-k_{\mathrm{c}}\right)}\left|\varphi_{G_{k}}\right\rangle \\
& =\frac{\sqrt{2 \pi}}{\alpha \sqrt{\Omega}} \sum_{k} \frac{\tilde{A}^{*}}{\sqrt{G_{k}}} e^{-i\left(k-k_{\mathrm{c}}\right) N_{\mathrm{c}}} e^{-\frac{\left(k-k_{\mathrm{c}}\right)^{2}}{2 \alpha^{2}}} e^{-i 2 \kappa t\left(k-k_{\mathrm{c}}\right)}\left|\varphi_{G_{k}}\right\rangle,
\end{aligned}
$$

then we substitute the eigen states $\left|\varphi_{G_{k}}\right\rangle=\sum_{j}\left(f_{j} / \sqrt{G_{k}}\right)|j\rangle$ in the expression of $|\psi(t)\rangle$, in the real space, we get

$$
\begin{aligned}
|\psi(t)\rangle \approx & \frac{\sqrt{2 \pi}}{\alpha \sqrt{\Omega}} \sum_{j=3}^{N} \sum_{k} \frac{\tilde{A}^{*}}{G_{k}} e^{-i\left(k-k_{\mathrm{c}}\right) N_{\mathrm{c}}} e^{-\frac{\left(k-k_{\mathrm{c}}\right)^{2}}{2 \alpha^{2}}} \\
& \times e^{-i 2 \kappa t\left(k-k_{\mathrm{c}}\right)} f_{j}|j\rangle \\
= & \sqrt{\frac{2 \sqrt{\pi}}{\alpha}} \sum_{j=3}^{N} \sum_{k} \frac{\tilde{A}^{*} B}{G_{k}} e^{-i k j} e^{-i\left(k-k_{\mathrm{c}}\right) N_{\mathrm{c}}} e^{-\frac{\left(k-k_{\mathrm{c}}\right)^{2}}{2 \alpha^{2}}} \\
& \times e^{-i 2 \kappa t\left(k-k_{\mathrm{c}}\right)}|j\rangle
\end{aligned}
$$

$$
\begin{aligned}
& +\sqrt{\frac{2 \sqrt{\pi}}{\alpha}} \sum_{j=3}^{N} \sum_{k} \frac{\tilde{A}^{*} A}{G_{k}} e^{i k j} e^{-i\left(k-k_{\mathrm{c}}\right) N_{\mathrm{c}}} e^{-\frac{\left(k-k_{\mathrm{c}}\right)^{2}}{2 \alpha^{2}}} \\
& \times e^{-i 2 \kappa t\left(k-k_{\mathrm{c}}\right)}|j\rangle,
\end{aligned}
$$

for the centerpart of the system (from $j=3$ to $j=N$ ). The time evolution of the Gaussian wave packet $|\psi(t)\rangle$ is reduced to a combination of two parts. After simplification, we note that before the Gaussian wave packet reaching the gain site of the $\mathcal{P} \mathcal{T}$-symmetric system, Eq. (B17) dominates and approximately equals to the time evolution of the Gaussian wave packet on a uniform chain; after the Gaussian wave packet being reflected at the gain site $\left[t>t_{0} \approx\left(N-N_{\mathrm{c}}\right) /(2 \kappa)\right]$, Eq. (B16) represents a persistent wave emission and Eq. (B17) stands for a reflected wave packet. The platform height $h=$ $2\left(\gamma_{\mathrm{c}} / \kappa\right)^{2} \sqrt{\pi} / \alpha$ shown in Eq. (17) is obtained after simplifying Eq. (B16), this persistent wave emission part results in a linear increasing of the initial state probability. As long as the system is at critical $\gamma_{\mathrm{c}}$, the linear increasing of the probability is visible even if the gain is small. Moreover, when $g^{4} / \kappa^{4} \gg 2 \alpha^{2} / \pi$, the wave emission dominates in the time evolution process. $|\psi(t)\rangle$ is approximately described by a platform wave emission, as

$$
\begin{aligned}
|\psi(t)\rangle & \approx \sqrt{\frac{2 \sqrt{\pi}}{\alpha}} \sum_{j=3}^{N} \sum_{k} \frac{\tilde{A}^{*} B}{G_{k}} e^{-i k j} e^{-i\left(k-k_{\mathrm{c}}\right) N_{\mathrm{c}}} e^{-\frac{\left(k-k_{\mathrm{c}}\right)^{2}}{2 \alpha^{2}}} e^{-i 2 \kappa t\left(k-k_{\mathrm{c}}\right)}|j\rangle \\
& \approx \frac{1}{4}\left(\frac{g}{\kappa}\right)^{2} \sqrt{\frac{2 \sqrt{\pi}}{\alpha}} \sum_{j=3}^{N}\left[\sum_{k} \frac{i(N-2)^{-1}}{\left(k-k_{\mathrm{c}}\right)} e^{4 i k} e^{-i\left(k-k_{\mathrm{c}}\right)\left(N_{\mathrm{c}}+2 \kappa t\right)} e^{-\frac{\left(k-k_{\mathrm{c}}\right)^{2}}{2 \alpha^{2}}} e^{-i k j}-e^{-i k_{\mathrm{c}} j}\right]|j\rangle \\
& =\frac{1}{2} \sqrt{\frac{2 \sqrt{\pi} \gamma_{\mathrm{c}}^{2}}{\alpha \kappa^{2}}} \sum_{j=3}^{N}\left[\sum_{k} i \frac{(N-2)^{-1}}{\left(k-k_{\mathrm{c}}\right)} e^{-\frac{\left(k-k_{\mathrm{c}}\right)^{2}}{2 \alpha^{2}}} e^{-i\left(k-k_{\mathrm{c}}\right)\left(j+N_{\mathrm{c}}+2 \kappa t-4\right)}-1\right] e^{-i k_{\mathrm{c}} j}|j\rangle \\
& =\frac{\sqrt{h}}{2} \sum_{j=3}^{N}\left[\sum_{k} i \frac{(N-2)^{-1}}{\left(k-k_{\mathrm{c}}\right)} e^{-\frac{\left(k-k_{\mathrm{c}}\right)^{2}}{2 \alpha^{2}}-i\left(k-k_{\mathrm{c}}\right)\left(j+N_{\mathrm{c}}+2 \kappa t-4\right)}-1\right] e^{-i k_{\mathrm{c}} j}|j\rangle \\
& \approx-\frac{\sqrt{h}}{2} \sum_{j=3}^{N}\left\{\operatorname{erf}\left(\frac{2^{3 / 4}}{\Delta}\left[2(\kappa t-N-2)+j+N_{\mathrm{c}}\right]\right)+1\right\} e^{-\frac{i \pi j}{2}}|j\rangle \\
& =-\frac{\sqrt{h}}{2} \sum_{j=3}^{N}\left\{\operatorname{erf}\left[\frac{2^{3 / 4}}{\Delta}\left(j-N_{t}\right)\right]+1\right\} e^{-\frac{i \pi j}{2}}|j\rangle,
\end{aligned}
$$

where $N_{t}=2(N+2)-N_{\mathrm{c}}-2 \kappa t$ indicates the Gaussian wave packet center after reflection for system with $\gamma=$ 0 . In the summation of $k, k \approx n \pi / N$ with integer $n=$ $1,2, \ldots, N$. The time evolution state $|\psi(t)\rangle$ at the end with gain is $|\psi(N+1, t)\rangle=-\left(\sqrt{2} i \gamma \kappa / g^{2}\right)|\psi(N, t)\rangle$, and $|\psi(N+2, t)\rangle=-(\sqrt{2} \kappa / g)|\psi(N, t)\rangle$. In the $\mathcal{P} \mathcal{T}$-symmetric system described by Hamiltonian in Eq. (19), the total site number of the system is $N+2$. However, the time evolution discussed in Sec. IV is for a system with gain only at one end, the total site number is $N+1$. In that case, therefore, we obtain the time evolution of the Gaussian wave packet in the wave emission system as shown in Eq. (16).
[1] A. Guo, G. J. Salamo, D. Duchesne, R. Morandotti, M. VolatierRavat, V. Aimez, G. A. Siviloglou, and D. N. Christodoulides, Phys. Rev. Lett. 103, 093902 (2009).
[2] S. Longhi, Opt. Lett. 40, 1278 (2015).

[3] X. Q. Li, X. Z. Zhang, G. Zhang, and Z. Song, Phys. Rev. A 91, 032101 (2015). 
[4] C. M. Bender and S. Boettcher, Phys. Rev. Lett. 80, 5243 (1998).

[5] F. G. Scholtz, H. B. Geyer, and F. J. W. Hahne, Ann. Phys. (NY) 213, 74 (1992).

[6] C. M. Bender, S. Boettcher, and P. N. Meisinger, J. Math. Phys. 40, 2201 (1999).

[7] C. M. Bender, D. C. Brody, and H. F. Jones, Phys. Rev. Lett. 89, 270401 (2002).

[8] A. de Souza Dutra, M. B. Hott, and V. G. C. S. dos Santos, J. Phys. A 34, L391 (2001).

[9] P. Dorey, C. Dunning, and R. Tateo, J. Phys. A 34, 5679 (2001).

[10] A. Mostafazadeh, J. Math. Phys. 43, 205 (2002).

[11] A. Mostafazadeh, J. Math. Phys. 43, 2814 (2002).

[12] A. Mostafazadeh, J. Math. Phys. 43, 3944 (2002).

[13] A. Mostafazadeh and A. Batal, J. Phys. A 36, 7081 (2003).

[14] A. Mostafazadeh and A. Batal, J. Phys. A 37, 11645 (2004).

[15] A. Mostafazadeh, J. Phys. A: Math. Gen. 39, 13495 (2006).

[16] H. F. Jones, J. Phys. A 38, 1741 (2005).

[17] L. Jin and Z. Song, Phys. Rev. A 80, 052107 (2009).

[18] L. Jin and Z. Song, Phys. Rev. A 81, 032109 (2010).

[19] A. Mostafazadeh, Phys. Rev. A 80, 032711 (2009).

[20] A. Mostafazadeh, Phys. Rev. A 84, 023809 (2011).

[21] A. Mostafazadeh, Phys. Rev. Lett. 110, 260402 (2013).

[22] A. Mostafazadeh and M. Sarisaman, Phys. Rev. A 87, 063834 (2013).

[23] A. Mostafazadeh and M. Sarisaman, Phys. Rev. A 88, 033810 (2013).

[24] S. Longhi, Phys. Rev. B 80, 165125 (2009).

[25] A. A. Andrianov, F. Cannata, and A. V. Sokolov, J. Math. Phys. 51, 052104 (2010).

[26] F. Correa and M. S. Plyushchay, Phys. Rev. D 86, 085028 (2012).

[27] L. Chaos-Cador and G. García-Calderón, Phys. Rev. A 87, 042114 (2013).

[28] A. Mostafazadeh, Phys. Rev. Lett. 102, 220402 (2009).

[29] H. F. Jones, Phys. Rev. D 76, 125003 (2007).

[30] H. F. Jones, Phys. Rev. D 78, 065032 (2008).

[31] M. Znojil, Phys. Rev. D 78, 025026 (2008).

[32] M. Znojil, Phys. Rev. D 80, 045009 (2009).

[33] M. Znojil, Phys. Rev. D 80, 045022 (2009).

[34] M. Znojil, Phys. Rev. D 80, 105004 (2009).

[35] C. M. Bender and P. D. Mannheim, Phys. Rev. D 78, 025022 (2008).

[36] S. Longhi, Phys. Rev. A 81, 022102 (2010).

[37] A. Ghatak, J. A. Nathan, B. P. Mandal, and Z. Ahmed, J. Phys. A: Math. Theor. 45, 465305 (2012).

[38] A. Mostafazadeh, Phys. Rev. A 87, 063838 (2013).
[39] X. Z. Zhang, L. Jin, and Z. Song, Phys. Rev. A 87, 042118 (2013).

[40] X. Z. Zhang and Z. Song, Ann. Phys. 339, 109 (2013).

[41] S. Longhi, Europhys. Lett. 106, 34001 (2014).

[42] G. R. Li, X. Z. Zhang, and Z. Song, Ann. Phys. 349, 288 (2014).

[43] S. Longhi, D. Gatti, and G. Della Valle, Sci. Rep. 5, 13376 (2015).

[44] S. Longhi, D. Gatti, and G. Della Valle, Phys. Rev. B 92, 094204 (2015).

[45] W. D. Heiss, J. Phys. A: Math. Theor. 45, 444016 (2012).

[46] I. Rotter, J. Phys. A: Math. Theor. 42, 153001 (2009).

[47] I. Rotter and A. F. Sadreev, Phys. Rev. E 71, 036227 (2005).

[48] Z. Ahmed, J. Phys. A: Math. Theor. 42, 472005 (2009).

[49] H. Ramezani, H.-K. Li, Y. Wang, and X. Zhang, Phys. Rev. Lett. 113, 263905 (2014).

[50] A. Mostafazadeh and H. Mehri-Dehnavi, J. Phys. A 42, 125303 (2009).

[51] A. Mostafazadeh, J. Phys. A: Math. Theor. 44, 375302 (2011).

[52] W. D. Heiss, H. Cartarius, G. Wunner, and J. Main, J. Phys. A: Math. Theor. 46, 275307 (2013).

[53] K. J. Vahala, Nature 424, 839 (2003).

[54] D. K. Armani, T. J. Kippenberg, S. M. Spillane, and K. J. Vahala, Nature 421, 925 (2003).

[55] B. Peng, S. K. Özdemir, F. Lei, F. Monifi, M. Gianfreda, G. L. Long, S. Fan, F. Nori, C. M. Bender, and L. Yang, Nat. Phys. 10, 394 (2014).

[56] B. Peng, S. K. Özdemir, S. Rotter, H. Yilmaz, M. Liertzer, F. Monifi, C. M. Bender, F. Nori, and L. Yang, Science 346, 328 (2014)

[57] Y. D. Chong, L. Ge, H. Cao, and A. D. Stone, Phys. Rev. Lett. 105, 053901 (2010).

[58] Y. Sun, W. Tan, H.-Q. Li, J. Li, and H. Chen, Phys. Rev. Lett. 112, 143903 (2014).

[59] W. Wan, Y. Chong, L. Ge, H. Noh, A. D. Stone, and H. Cao, Science 331, 889 (2011).

[60] H. F. Jones, arXiv:1106.1855 (2011).

[61] W. Kim, L. Covaci, and F. Marsiglio, Phys. Rev. B 74, 205120 (2006).

[62] E. M. Graefe, H. J. Korsch, and A. E. Niederle, Phys. Rev. A 82, 013629 (2010).

[63] E. M. Graefe, H. J. Korsch, and A. E. Niederle, Phys. Rev. Lett. 101, 150408 (2008).

[64] H. Cartarius and N. Moiseyev, Phys. Rev. A 84, 013419 (2011).

[65] W. D. Heiss, Eur. Phys. J. D 60, 257 (2010). 